\title{
Dynamical signatures of the vulcanization transition
}

\author{
Kurt Broderix, Paul M. Goldbart, and Annette Zippelius ${ }^{1}$ \\ ${ }^{1}$ Institut für Theoretische Physik, Universität Göttingen, D-37073 Göttingen, Germany \\ ${ }^{2}$ Department of Physics, University of Illinois at Urbana-Champaign, 1110 West Green Street, Urbana, Illinois 61801, USA
}

(August 13, 1997)

\begin{abstract}
Dynamical properties of vulcanized polymer networks are addressed via a Rouse-type model that incorporates the effect of permanent random crosslinks. The incoherent intermediate scattering function is computed in the sol and gel phases, and at the vulcanization transition between them. At any nonzero crosslink density within the sol phase Kohlrausch relaxation is found. The critical point is signalled by divergence of the longest time-scale, and at this point the scattering function decays algebraically, whereas within the gel phase it acquires a time-persistent part identified with the gel fraction.
\end{abstract}

PACS numbers: $64.60 . \mathrm{Ht}, 82.70 . \mathrm{Gg}, 61.43 . \mathrm{Fs}$

Vulcanized matter has been suggested as a model substance for the transition from a viscous fluid to an amorphous solid state, i.e. the glass transition. Experimentally one has access to the static and dynamic critical behavior, and theoretically a reliable semi-microscopic model is available for actually computing properties of the system in the amorphous state as well as close to the glass transition. So far, the approach based on statistical mechanics has mainly focused on static properties. Here we extend the analysis to dynamic quantities and discuss the motion of a tagged monomer. We thereby show that vulcanized systems display many of the dynamic characteristics of glassy systems, in particular a Kohlrausch law (generalized to nonzero wavenumbers) in the fluid phase and a divergence of the longest time-scale as the glass transition is approached.

The gelation transition has been discussed very successfully in terms of a percolation transition [1]. The percolation threshold is identified with the critical crosslink concentration, and the average cluster size with the weight average molecular weight in the sol phase. The percolation picture has been confirmed by a statisticalmechanical approach 20.4. In addition, the latter approach allows one to discuss structural and elastic properties of the gel phase, which can not be obtained from a percolation model unless additional assumptions are made. The shortcomings of percolation theory become even more apparent if one is interested in dynamic quantities. There is obviously no dynamic percolation model that could account for the observed anomalous relaxation while approaching the gelation transition from the sol phase.

Here we start from a (semi-)microscopic equation of motion, the Rouse model 5], and compute the incoherent intermediate scattering function as the vulcanization transition is approached from the liquid side. We only consider permanently formed crosslinks and model them by a harmonic potential. We furthermore assume that each polymer is equally likely to be crosslinked to each other polymer. We do not distinguish between gelation and vulcanization and use sol phase and fluid phase as synonyms, because, as we shall see, the long time dynamics is not affected by the molecular size and structure of the building blocks of the random network. Neglecting excluded volume interactions, we analyse the dynamics by making use of the theory of random graphs, created by Erdös and Rényi [6]. (For an informal account of random graph theory see Ref. [8].) Our main results are the following:

- We observe three distinct phases with qualitatively different long-time dynamics.

- If the number of crosslinks $M$ is submacroscopic (i.e. $o(N)$, where $N$ is the number of macromolecular chains in the uncrosslinked system) then the long-time behavior of the autocorrelation is diffusive: $S_{t}(\boldsymbol{q}) \propto$ $\exp \left\{-D_{0} \boldsymbol{q}^{2} t\right\}$.

- If the crosslink concentration $c:=M / N$ is non-zero but smaller than the critical value $c_{\text {crit }}$ for vulcanization then the time-decay of the autocorrelation is characterized by a spectrum of relaxation times, reflecting the diffusive motion of a distribution of cluster sizes. The autocorrelation decays for large times as a stretched exponential: $S_{t}(\boldsymbol{q}) \propto \exp \left\{-\left(t / \tau_{\boldsymbol{q}}\right)^{1 / 2}\right\}$ with, however, a scaling of time with wavenumber that is specific to a diffusion process: $\tau_{\boldsymbol{q}}^{-1} \propto D_{0} \boldsymbol{q}^{2}$. This relaxation is the true asymptotic behavior, and should not be confused with a formally similar time decay in the Rouse model for uncrosslinked systems, which occurs for time-scales smaller than the Rouse time [5].

- As the vulcanization transition is approached, i.e. for $c \uparrow c_{\text {crit }}$, the longest time-scale diverges like the inverse squared distance from the critical point, whereas the generalized diffusion constant goes to zero linearly and, more generally, is proportional to the weight average molecular weight.

- Precisely at $c_{\text {crit }}$ the distribution of relaxation times falls off algebraically for large relaxation times, implying a $\left(D_{0} \boldsymbol{q}^{2} t\right)^{-1 / 2}$ decay of the autocorrelation.

- In the gel phase, i.e. for $c>c_{\text {crit }}$, the autocorrelation acquires a time-persistent part, which is identical to the gel fraction as obtained from static calculations.

- The mean square displacement grows linearly with 
time for all crosslink concentrations, with only a weak singularity at the vulcanization transition. Its long-time behavior is dominated by the "mobile" monomers on finite clusters, even in the gel phase.

We consider a system of $N$ linear, identical, monodisperse chains of arc-length $L$ with force-free ends. Monomer $s$ on chain $i$ is characterized by its timedependent position vector $\boldsymbol{R}_{t}(i, s)(i=1, \ldots, N$ and $0 \leq s \leq L)$ in $d$-dimensional space. The simplest dynamics is purely relaxational:

$$
\partial_{t} \boldsymbol{R}_{t}(i, s)=-\frac{1}{\zeta} \frac{\delta H}{\delta \boldsymbol{R}_{t}(i, s)}+\boldsymbol{\eta}_{t}(i, s) .
$$

The monomers' relaxation to the stationary state $\delta H / \delta \boldsymbol{R}=\mathbf{0}$ with rate $\zeta$ is perturbed by thermal fluctuations, which are modeled as Gaussian white noise $\boldsymbol{\eta}$ with zero mean and variance $\left\langle\boldsymbol{\eta}_{t}^{\nu}(i, s) \boldsymbol{\eta}_{t^{\prime}}^{\mu}\left(i^{\prime}, s^{\prime}\right)\right\rangle=$ $2 \zeta^{-1} \delta^{\nu, \mu} \delta\left(t-t^{\prime}\right) \delta_{i, i^{\prime}} \delta\left(s-s^{\prime}\right)$, where $\nu, \mu=1, \ldots, d$. We measure energies in units such that $k_{B} T$ is unity. If the Hamiltonian were to consist only of the Wiener term

$$
H_{W}:=\frac{d}{2 l} \sum_{i=1}^{N} \int_{0}^{L} \mathrm{~d} s\left(\frac{\partial \boldsymbol{R}_{t}(i, s)}{\partial s}\right)^{2}
$$

then the dynamical equation would reduce to the Rouse model. Here $l$ denotes the persistence length of the individual polymers. In the following we wish to treat a crosslinked melt or solution. We only consider the case of permanently formed crosslinks that constrain randomly chosen pairs of monomers, and we study the relaxation of monomers in the presence of a fixed crosslink configuration. A particular realization of $M$ crosslinks is characterized by a set of indices $\left\{i_{e}, s_{e} ; i_{e}^{\prime}, s_{e}^{\prime}\right\}_{e=1}^{M}$ specifying which monomer is connected to which other monomer. We adopt here the simplest distribution of crosslinks, i.e. the crosslinks are independent of each other and for each crosslink the pair $\left(i_{e} ; i_{e}^{\prime}\right)$ of polymer indices is realized with equal probability. We leave unspecified the distribution of monomer labels $\left(s_{e} ; s_{e}^{\prime}\right)$ because its form will not affect our results. Thus, for instance, the case of end-linking with unconstrained functionality is included. The crosslinks are modeled by a harmonic potential

$$
U:=\frac{d}{2 a^{2}} \sum_{e=1}^{M}\left(\boldsymbol{R}_{t}\left(i_{e}, s_{e}\right)-\boldsymbol{R}_{t}\left(i_{e}^{\prime}, s_{e}^{\prime}\right)\right)^{2},
$$

where $a>0$ controls the distance between monomers within a crosslink. The Hamiltonian that we use is thus $H:=H_{W}+U$. Hard $\delta$-constraints for the crosslinks can be recovered from the harmonic potential in the limit $a \rightarrow 0$ [9].

The quantity of interest is the intermediate incoherent scattering function

$$
\begin{aligned}
S_{t}(\boldsymbol{q}):= & \lim _{t_{o} \rightarrow \infty} \frac{1}{N} \sum_{i=1}^{N} \int_{0}^{L} \frac{\mathrm{d} s}{L} \\
& \left\langle\exp \left\{i \boldsymbol{q} \cdot\left(\boldsymbol{R}_{t+t_{o}}(i, s)-\boldsymbol{R}_{t_{o}}(i, s)\right)\right\}\right\rangle .
\end{aligned}
$$

The above average refers to the noise $\boldsymbol{\eta}$ and is taken with fixed given crosslink configuration.

As the Hamiltonian is quadratic the Langevin equation (11) is linear and $\boldsymbol{R}$ is a Gaussian Markov process, whose distribution is in the limit $t_{o} \rightarrow \infty$ characterized by

$$
\begin{gathered}
\frac{1}{2 d}\left\langle\left(\boldsymbol{R}_{t+t_{o}}(i, s)-\boldsymbol{R}_{t_{o}}(i, s)\right)\left(\boldsymbol{R}_{t+t_{o}}\left(i^{\prime}, s^{\prime}\right)-\boldsymbol{R}_{t_{o}}\left(i^{\prime}, s^{\prime}\right)\right)\right\rangle \\
=\frac{1}{\zeta} \int_{0}^{t} \mathrm{~d} \tau \mathrm{e}^{-\tau \Gamma}\left(i, s ; i^{\prime}, s^{\prime}\right)=: G_{t}\left(i, s ; i^{\prime}, s^{\prime}\right) .
\end{gathered}
$$

Here $\mathrm{e}^{-\tau \Gamma}\left(i, s ; i^{\prime}, s^{\prime}\right)$ is the matrix representation of the exponential of the linear operator $\Gamma$ which stems from the deterministic part of Eq. (1) and is defined by its action, viz.,

$$
\begin{aligned}
(\Gamma f)(i, s):=-\frac{d}{\zeta l} \frac{\partial^{2}}{\partial s^{2}} f(i, s) & \\
+\frac{d}{\zeta a^{2}} \sum_{e=1}^{M}\left(\delta_{i, i_{e}} \delta\right. & \left.\left(s-s_{e}\right)-\delta_{i, i_{e}^{\prime}} \delta\left(s-s_{e}^{\prime}\right)\right) \\
& \times\left(f\left(i_{e}, s_{e}\right)-f\left(i_{e}^{\prime}, s_{e}^{\prime}\right)\right),
\end{aligned}
$$

on functions $f$ over the configuration space obeying Neumann boundary-conditions with respect to $s$.

We are only concerned with the long-time behavior of the scattering function. Thus we need the asymptotics of $G_{t}$ as $t \rightarrow \infty$, which (because $\Gamma$ is non-negative definite by inspection) is governed by the eigenspace of $\Gamma$ corresponding to zero eigenvalues. Moreover, $\Gamma f=0$ implies $(f, \Gamma f)=0$, and this in turn implies (by virtue of Eq. (6) and the boundary conditions) that both $\partial f(i, s) / \partial s=0$ for all $i=1, \ldots, N$ and $f\left(i_{e}, s_{e}\right)=f\left(i_{e}^{\prime}, s_{e}^{\prime}\right)$ for all $e=1, \ldots, M$. Let us group the polymers into $K$ clusters $\left\{\mathcal{N}_{k}\right\}_{k=1}^{K}$, which are defined as maximal path-wise connected components. Then, according to the above reasoning, the eigenspace in question consists of functions that are constant when restricted to any one cluster. We therefore have, as $t \rightarrow \infty$,

$$
G_{t}\left(i, s ; i^{\prime}, s^{\prime}\right)=D_{0} t \sum_{k=1}^{K} \frac{1}{N_{k}} \delta_{\mathcal{N}_{k}}(i) \delta_{\mathcal{N}_{k}}\left(i^{\prime}\right)+\mathcal{O}(1),
$$

with the bare diffusion constant $D_{0}=1 /(\zeta L)$. Here $N_{k}$ denotes the number of polymers in the $k$-th cluster $\mathcal{N}_{k}$, whence $\sum_{k=1}^{K} N_{k}=N$, and $\delta_{\mathcal{N}_{k}}(i)=1$ if $i \in \mathcal{N}_{k}$ and $\delta_{\mathcal{N}_{k}}(i)=0$ otherwise.

Due to the Gaussian nature of the process $\boldsymbol{R}$ we can express the scattering function in terms of $G$, and find for the long-time behavior of the scattering function

$$
S_{t}(\boldsymbol{q})=\frac{1}{N} \sum_{k=1}^{K} N_{k} \exp \left\{-\boldsymbol{q}^{2}\left(\frac{D_{0} t}{N_{k}}+\mathcal{O}_{k}(1)\right)\right\} .
$$

We have equipped the long-time corrections $\mathcal{O}_{k}(1)$ with the cluster index $k$ to indicate that we are not allowed to handle them as an overall pre-factor. 
The above result (8) is not at all surprising. It merely confirms the notion that the slowest modes correspond to the center-of-mass diffusion of individual polymer clusters. Here $D_{0} / N_{k}$ may be interpreted as the diffusion constant of the $k$-th cluster, and $N_{k} / N$ as the probability for a given site to belong to the $k$-th cluster. We remark that in accordance with this notion the strength of the crosslink potential does not enter in Eq. (8). Thus the result covers the limiting case, $a \rightarrow 0$, of hard crosslinks, too.

The statistics of the polymer clusters' sizes is determined by the crosslink distribution. Two different polymers are considered to be linked if there is at least one crosslink joining any two monomers of the respective polymers. In our model we specify the total number of crosslinks $M$ so that the total number of polymerpolymer links varies in different crosslink realizations. These fluctuations are suppressed [10] in the macroscopic limit as there are $\mathcal{O}\left(N^{2}\right)$ possibilities for $M=\mathcal{O}(N)$ crosslinks to form $M$ different polymer-polymer links and only $\mathcal{O}(N)$ other possibilities. For any given number of polymer-polymer links, the statistics of the clusters' sizes can be taken over from Ref. [6] by identifying polymer clusters with components of random graphs.

As shown in Ref. [6], the polymer clusters exhibit the analogue of a percolation transition. Below the crosslink concentration $c_{\text {crit }}=1 / 2$ there will be no clusters of macroscopic size and almost all polymers are members of tree clusters 11, which are defined by the absence of loops. For $c>\frac{1}{2}$ there will be $\mathcal{O}(N)$ polymers in exactly one macroscopic cluster, which is not a tree, and almost all other polymers still belong to tree clusters of submacroscopic size [12]. Let $T_{n}$ denote the average number of tree-clusters consisting of $n$ polymers. Then, whatever the crosslink concentration $c>0$ may be, one has 13

$$
\lim _{N \rightarrow \infty} \frac{1}{N} T_{n}=\tau_{n}=\frac{1}{2 c} \frac{n^{n-2}}{n !}\left(2 c \mathrm{e}^{-2 c}\right)^{n} .
$$

In the macroscopic limit, the average fraction $\mathcal{S}_{\infty}$ of polymers in the giant cluster is given by [14.

$$
\mathcal{S}_{\infty}=1-\sum_{n=1}^{\infty} n \tau_{n}
$$

which, for $c \leq \frac{1}{2}$, amounts to $\mathcal{S}_{\infty}=0$ 1.

To work out the long-time behavior of the autocorrelation, given the statistics of polymer clusters' sizes, we shall first make the simplifying assumption that we can ignore the possible dependence of the long-time correction $\mathcal{O}_{k}(1)$ on the index $k$ of the polymer cluster. This assumption can indeed be justified 10 for finite cluster sizes. From Eqs. (8) and (9) we get for the scattering function, averaged over the distribution of crosslinks

$$
S_{t}(\boldsymbol{q})=\mathcal{S}_{\infty}+\sum_{n=1}^{\infty} n \tau_{n} \exp \left\{-D_{0} \boldsymbol{q}^{2} t / n\right\}
$$

This result holds for all $c>0$ with $\mathcal{S}_{\infty}=0$ for $0 \leq c \leq \frac{1}{2}$. In fact, one would expect $S_{t}(\boldsymbol{q})$ to be self-averaging [10].

We now discuss four distinct regimes:

- For a submacroscopic number of crosslinks there is only a submacroscopic number of clusters, which do not contribute in the thermodynamic limit. Hence the scattering function decays, to leading order, according to the Rouse model: $S_{t}(\boldsymbol{q})=\exp \left\{-D_{0} \boldsymbol{q}^{2} t\right\}$.

- In the sol phase with a non-zero concentration of crosslinks, i.e. for $0<c<\frac{1}{2}$, the distribution of cluster sizes falls off exponentially for large $n$ :

$$
n \tau_{n} \sim \frac{1}{2 c \sqrt{2 \pi}} n^{-3 / 2} \exp \{-n h(c)\}
$$

with $h(c):=2 c-1-\ln (2 c)>0$. This exponential distribution of cluster sizes - and hence diffusive time-scalesgives rise to a stretched exponential decay of the scattering function in the sol phase:

$$
S_{t}(\boldsymbol{q}) \sim \frac{1}{\left(8 c^{2} D_{0} \boldsymbol{q}^{2} t\right)^{1 / 2}} \exp \left\{-2\left(h(c) D_{0} \boldsymbol{q}^{2} t\right)^{1 / 2}\right\}
$$

for $D_{0} \boldsymbol{q}^{2} t \rightarrow \infty$. The time-scale of the stretched exponential diverges like $\left(c-\frac{1}{2}\right)^{-2}$ as the vulcanization transition is approached, whereas the generalized diffusion constant,

$$
D_{\text {eff }}^{-1}:=\lim _{\boldsymbol{q} \rightarrow 0} \boldsymbol{q}^{2} \int_{0}^{\infty} \mathrm{d} t S_{t}(\boldsymbol{q})=\frac{D_{0}^{-1}}{1-2 c},
$$

goes to zero linearly as $c \uparrow \frac{1}{2}$.

- At the critical point for vulcanization, i.e. at $c=$ $\frac{1}{2}$, the distribution of cluster sizes falls off algebraically, $n \tau_{n} \propto n^{-3 / 2}$. Consequently the autocorrelation shows algebraic decay in time: $S_{t}(\boldsymbol{q}) \propto\left(D_{0} \boldsymbol{q}^{2} t\right)^{-1 / 2}$.

- In the gel phase, i.e. for $c>\frac{1}{2}$, the scattering function acquires a time-persistent part $\mathcal{S}_{\infty}$, which is given 15 by the largest root of $1-\mathcal{S}_{\infty}=\exp \left(-2 c \mathcal{S}_{\infty}\right)$. As expected, the time-persistent part of the autocorrelation is just the fraction of polymers in the macroscopic cluster, i.e. the gel fraction. The distribution (9) of finite cluster sizes is valid on both sides of the transition [16]. Hence we expect to see the stretched exponential behaviour (13) for the decaying part of the scattering function in the gel phase as well.

Lastly, we mention that the time-delayed square monomer displacement,

$$
C_{t}:=\frac{1}{N} \sum_{i=1}^{N} \int_{0}^{L} \mathrm{~d} s G_{t}(i, s ; i, s) \sim 2 D t,
$$

increases asymptotically linearly with time for all crosslink concentrations $c$. In terms of polymer clusters the proportionality constant $D$ is given by $D=$ $D_{0} K / N=D_{0} \sum_{n=1}^{\infty} \tau_{n}$. This changes smoothly with crosslink concentration: in the sol phase it is given by 
$D=D_{0}(1-c)$; at $c=1 / 2$ it is twice continuously differentiable; deep in the gel phase we find $D \sim D_{0} \exp (-2 c)$. Hence the squared monomer displacement is unsuitable for detecting the sol-gel transition because its long-time behavior is always dominated by the finite polymer clusters.

We conclude with a few remarks concerning the experimental situation. Relaxation following a stretched exponential $\exp \left\{-(t / \tau)^{\beta}\right\}$ seems to be a universal feature of glassy systems. It has been observed in fluids undergoing either thermal or chemical vitrification, as well as in magnetic systems undergoing a spin-glass transition. It can always be interpreted in terms of a distribution of time-scales, although the nature of the underlying elementary process may be quite different. In the present model the elementary dynamical process is diffusion, and the stretched exponential results from a superposition of many diffusive modes, reflecting the distribution of cluster sizes [17]. This gives rise to a highly characteristic $\boldsymbol{q}$ dependence of the time-scale of the stretched exponential, namely $\tau_{\boldsymbol{q}}^{-1} \propto D_{0} \boldsymbol{q}^{2}$. Such a time-wavenumber scaling has indeed been seen in neutron spin-echo experiments on inorganic and organic glasses 18].

As the gelation transition is approached one observes experimentally a divergence of the longest time-scale, and at the critical point density fluctuations are found to decay algebraically. We compare our results to the data of Martin et al. 19]. These authors use quasi-elastic light scattering to study silica sol-gels in the pre- and post-gel regimes. They observe a stretched exponential in the sol phase with an exponent $\beta \approx 0.66$ and, furthermore, relate the time-scale $\tau$ of the stretched exponential to a diffusive time scale. The latter is found to diverge as the gel point is approached with an exponent close to 2.2. In the critical gel they find algebraic decay of density fluctuations with an exponent value of $\approx 0.35$. All these findings agree qualitatively with the predictions of our model. In fact, our expression (8) for $S_{t}(\boldsymbol{q})$ has been suggested on phenomenological grounds as a starting point for a discussion of the critical dynamics at the sol-gel transition [20]. Although a general scaling of the diffusion constant of a individual cluster with its size was postulated, it has turned out that the so-called Rouse limit, which leads back to our representation (8), agrees well with experiment [19]. In comparing our results with experiment one should keep in mind that we have used a distribution of crosslinks lacking any correlations, and have not taken into account excluded volume interactions. Presumably this amounts to a mean-field approximation of a shortrange model. Vulcanization of a dense melt of linear macromolecules has been argued [21] to be mean-fieldlike, except within a narrow critical region of crosslink densities whose width decreases like the one third power of the ratio of the persistence length to the chain length. Since the argument includes and is in fact based on the distribution of cluster sizes, it should also hold for the dynamics.

Acknowledgments - We thank R. Kree for useful discussions. This work was supported by U.S. National Science Foundation Grant DMR94-24511 (PG), by NATO CRG 94090 (AZ,PG), and by the DFG through SFB 345 (AZ).

[1] P. G. de Gennes, Scaling Concepts in Polymer Science, (Cornell University Press, Ithaca, 1979); D. Stauffer, A. Coniglio and M. Adam, Adv. in Polym. Sci. 44 (1982) 103.

[2] R. T. Deam and S. F. Edwards, Phil. Trans. R. Soc. London A280 (1976) 317.

[3] R. C. Ball and S. F. Edwards, Macromol. 13 (1980) 748;

[4] P. M. Goldbart, H. Castillo and A. Zippelius, Adv. in Phys. 45 (1996) 393.

[5] M. Doi and S. F. Edwards, The Theory of Polymer Dynamics, (Clarendon Press, Oxford, 1985).

[6] P. Erdős and A. Rényi, Magyar Tud. Akad. Mat. Kut. Int. Közl. 5 (1960) 17; reprinted in Ref. [7], Chap. 14, article [324].

[7] P. Erdős: The Art of Counting, edited by J. Spencer (MIT Press, 1973).

[8] P. Erdős and A. Rényi, Bull. Inst. Internat. Statist. 38 (1961) 343; reprinted in Ref. [7], Chap. 14, article [v].

[9] M. P. Solf and T. A. Vilgis, J. Phys. A 28 (1995) 6655.

[10] K. Broderix, P. Goldbart, A. Zippelius, in preparation.

[11] This is a consequence of Theorem 5 d together with Theorem 5 e in Ref. [6].

[12] This follows from the combination of Theorem $9 \mathrm{~b}$ and Theorem 6 in Ref. [6].

[13] This distribution follows from Theorem $2 \mathrm{~b}$ in Ref. [6] and is identical to the one obtained from a mean-field theory of percolation, see M. J. Stephen in Critical phenomena, F. J. W. Hahne (Ed.) (Springer, Berlin, 1983).

[14] See Theorem 9b in Ref. 66.

[15] See Eqs. (4.4) and (4.5) in Ref. [6].

[16] This is a peculiarity of the random graph and mean-field description of percolation and is not valid in general, see Section 2.8 in D. Stauffer, Introduction to percolation theory (Taylor \& Francis, London, 1985).

[17] More generally, only the total length of all chains linked to a cluster determines the asymptotic dynamics. Hence our conclusions are not restricted to vulcanized chains but, e.g., hold equally well for polydisperse melts with a length distribution falling exponentially.

[18] F. Mezei, W. Knaak and B. Farago, Phys. Rev. Lett. 58 (1987) 571; D. Richter, B. Frick and B. Farago, Phys. Rev. Lett. 61 (1988) 2465.

[19] J. E. Martin, J. Wilcoxon and J. Odinek, Phys. Rev. A 43 (1991) 858. For a review see J. E. Martin and D. Adolf, Ann. Rev. Phys. Chem. 42 (1991) 311.

[20] P. G. de Gennes, J. Physique (Paris) 40 (1979) L-197; J. E. Martin and J. Wilcoxon, Phys. Rev. Lett. 61 (1988) 373.

[21] D. Stauffer, J. Chem. Soc. Faraday Trans. II, 72 (1976) 1354; P. G. de Gennes, J. Phys. Lett. (Paris) 38 (1977) L-355. 